\title{
Improved Magnetotelluric Zohdy-Oldenburg Direct Inversion
}

\author{
Hui Cao, ${ }^{1}$ Yun Liu, ${ }^{2}$ and Kun Peng Wang ${ }^{1}$ \\ ${ }^{1}$ Key Laboratory of Earth Exploration and Information Technology of MOE, Chengdu University of Technology, Chengdu, \\ Sichuan 610059, China \\ ${ }^{2}$ Institute of Geochemistry, Chinese Academy of Sciences, Guiyang, Guizhou 550002, China \\ Correspondence should be addressed to Yun Liu; liuyun_cdut@163.com
}

Received 1 August 2013; Accepted 4 December 2013

Academic Editor: Davide La Torre

Copyright (C) 2013 Hui Cao et al. This is an open access article distributed under the Creative Commons Attribution License, which permits unrestricted use, distribution, and reproduction in any medium, provided the original work is properly cited.

Based on researches of Baiyao (1995) and Zhang and Xu (2001), this paper proposes an improved 2D MT Zohdy-Oldenburg direct inversion method, in the least-square sense, embodying the features of Zohdy's ratio method and Oldenburg's difference method, in the condition of rugged topography, with phase information. It bypasses large calculations of the Jacobian matrix and large sparse linear systems of equations and enables direct modifications and comparisons of the model parameters. According to the calculation and analysis of examples, it shows faster convergence and higher precision. In contrast with the conventional linear inversion, the calculation speed of this new method can be increased by more than 10 times.

\section{Introduction}

Calculating Jacobian matrix and solving large systems of linear equations are required in conventional magnetotelluric (referred to later as MT) linear or nonlinear optimized inversion method [1-4]. It is imaginable that the calculation is very complicated and the systems of equations are often ill conditioned. Therefore in this paper we think it is necessary to find a quick and effective inversion method to solve practical problems in engineering projects.

Zohdy's [5] method for the inversion calculation of direct-current resistivity sounding method (1989) converts polar distance $(\mathrm{AB} / 2)$ to equivalent depth and modifies the model resistivity with the ratio between forward curve and measured curve to eliminate errors caused by zeroorder asymptotic inversion (ratio method). This is called the asymptotic iterative inversion. Oldenburg and Ellis [6] in 1991 also proposed an iterative inversion method for MT studies, which modifies the model resistivity and model depth with the difference, calculated from Bostick, of forward result data and measured data of the prediction model inversion (difference method). Zohdy's method is to modify the model resistivity with ratio method, so the rate of iterative convergence is apparently slower than that of Oldenburg's method. In Zohdy's iterative process, sometimes the model modification may be too much that the fitting errors will diverge. To solve this problem, two papers authored, respectively, by Shizhe and Bin (1995) $[7,8]$ suggest that one correction factor should be added to Zohdy's method to control the step size which modifies the model resistivity. This inversion method is named as the curve comparison. Baiyao (1995) [9] repeated his study on the methods used by Zohdy and Oldenburg and proposed a least-square-sense-based direct inversion method, which combines Zohdy's ratio method with Oldenburg's difference method by filling longitudinal conductivity in the model grid and applies the combination in the inversion of directcurrent resistivity sounding method [10] and MT sounding method [11]. On the basis of researches of Shizhe and Bin (1995) $[7,8]$ and Weidelt's MT impedance phase and apparent resistivity theory [12], Zhang and $\mathrm{Xu}[13,14]$ added impedance phase information to Zohdy's ratio method and thus improved the rate of convergence in its iterative process.

This shows that the MT direct inversion, bypassing calculating Jacobian matrix and solving large systems of linear equations, directly modifying and providing comparisons for the model parameters, can fully rival Bostick inversion. Therefore, to solve practical problems like slow speed of inversion and poor fitting precision found in engineering projects, this paper, based on previous studies, aims to 
improve the direct inversion method, namely, to propose an improved 2D MT Zohdy-Oldenburg direct inversion method with phase information.

\section{Improved Zohdy-Oldenburg Direct Inversion in the Least-Square Sense}

Suppose that there are $N$ parameters of a geoelectric prediction model, and they are $\mathbf{m}=\left(m_{1}, m_{2}, \ldots, m_{N}\right)^{T}$. Their relationship with surface observed values, $M_{a}$, is as follows:

$$
M_{a}=M_{c}(\mathbf{m})+O(\mathbf{m}) .
$$

In this equation, $M_{c}(\mathbf{m})$ means theoretical observed values obtained from the forward computation of $N$ model parameters, and $O(\mathbf{m})$ means errors between observed values and theoretical values.

To obtain the result $O(\mathbf{m}) \approx 0$, that is to say, the errors between observed values $M_{a}$ and theoretical values $M_{c}(\mathbf{m})$ can reach a certain degree of precision, multiple iterations and modifications of $\mathbf{m}$, parameters of the geoelectric model, are often needed before a group of optimized $\mathbf{m}$ are produced. This is the general process of geophysical inversion.

During the $k$ th iterative calculation, for the prediction model $\mathbf{m}^{(k)}$, we expand (1) to Taylor series and neglect terms which contain higher than second power in $\Delta m_{j}^{(k)}=m_{j}-$ $m_{j}^{(k)}$, and then we get

$$
M_{a}=M_{c}\left(\mathbf{m}^{(k)}\right)+\sum_{j=1}^{N} \frac{\partial M_{c}\left(\mathbf{m}^{(k)}\right)}{\partial m_{j}^{(k)}} \Delta m_{j}^{(k)} .
$$

When there are $M$ observed data, in matrix form, the above equation can be put into

$$
\mathbf{M}_{a}=\mathbf{M}_{c}^{(k)}+\mathbf{A}^{(k)} \Delta \mathbf{m}^{(k)},
$$

where $\mathbf{A}^{(k)}$ is the $M \times N$ matrix, the Jacobian matrix (partial derivative matrix).

Establishing the objective function is often needed in conventional linear inversions:

$$
\varphi(\mathbf{m})=\left\|\mathbf{M}_{a}-\left(\mathbf{M}_{c}^{(k)}+\mathbf{A}^{(k)} \Delta \mathbf{m}^{(k)}\right)\right\|^{\alpha}
$$

where $\alpha$ is the matrix norm, and when $\alpha$ is 2 , it becomes the least-square method. To make the objective function $\varphi(\mathbf{m})$ tend to minimum and thus determine the amount of the model parameters' modification $\Delta \mathbf{m}$, calculating the Jacobian matrix and solving systems of linear equations are needed. This kind of inversion is burdened with large calculation and often contains ill-conditioned systems of equations.

To avoid this problem, we introduce the ZohdyOldenburg direct inversion method, which can also be called the asymptotic iterative method. If we replace the $\mathbf{A}^{(k)}$ in the Jacobian matrix with $\mathbf{B}^{(k)}$, the objective function in (4) becomes

$$
\varphi(\mathbf{m})=\left\|\mathbf{M}_{a}-\left(\mathbf{M}_{c}^{(k)}+\mathbf{B}^{(k)} \Delta \mathbf{m}^{(k)}\right)\right\|^{\alpha}
$$

And when we use the second-order norm, this turns into a question about the least-square method, and its solution is

$$
\Delta \mathbf{m}^{(k)}=\mathbf{W}^{(k)}\left(\mathbf{M}_{a}-\mathbf{M}_{c}^{(k)}\right)
$$

where $\mathbf{W}^{(k)}=\left(\mathbf{B}^{(k)}\right)^{-1}$ and $\Delta \mathbf{m}^{(k)}=\mathbf{m}^{(k+1)}-\mathbf{m}^{(k)}$ and therefore the following equation can be derived:

$$
\mathbf{m}^{(k+1)}=\mathbf{m}^{(k)}+\mathbf{W}^{(k)}\left(\mathbf{M}_{a}-\mathbf{M}_{c}^{(k)}\right) .
$$

To keep the algorithm from converging, a correction factor $c(c \leq 1)$ will be added to $(7)$ to limit the step size of modification. Thus we can obtain the iterative formula of Zohdy-Oldenburg direct inversion method in the leastsquare sense.

In this case, when it comes to the $j$ th model parameter, the inversion formula for $(k+1)$ th iterative calculation is

$$
m_{j}^{(k+1)}=m_{j}^{(k)}+c W_{j}^{(k)}\left(M_{a j}-M_{c j}^{(k)}\right) .
$$

When $c=W_{j}=1$, (8) becomes Oldenburg's asymptotic iterative method, which modifies the model with the difference method.

When $W_{j}^{(k)}=m_{j}^{(k)} / M_{c j}^{(k)},(8)$ turns into

$$
m_{j}^{(k+1)}=m_{j}^{(k)}+c\left(\frac{M_{a j}}{M_{c j}^{(k)}}-1\right) m_{j}^{(k)} .
$$

If $c=1$, (9) turns into Zohdy's asymptotic iterative method, which modifies the model with the ratio method.

Hence we can conclude that the improved ZohdyOldenburg direct inversion has features of asymptotic iterative method of the two scholars, ensuring that the algorithm in the iterative process can achieve faster convergence and higher stability of data.

\section{Two-Dimensional Improved Zohdy- Oldenburg Direct Inversion}

3.1. Two-Dimensional Direct Inversion. In the 2D inverse modeling of MT, we adopt an improved Zohdy-Oldenburg direct inversion method. The geoelectric prediction model's parameter, $\mathbf{m}=\left(m_{1}, m_{2}, \ldots, m_{N}\right)^{T}$, is divided into two parts, the resistivity $\boldsymbol{\rho}=\left(\rho_{1}, \rho_{2}, \ldots, \rho_{M}\right)^{T}$ and the depth $\mathbf{h}=\left(h_{1}, h_{2}, \ldots, h_{M}\right)^{T}$. So (9) can be changed to the MT 2D improved Zohdy-Oldenburg direct inversion iterative formula:

$$
\begin{aligned}
& \rho_{i, j}^{(k+1)}=\rho_{i, j}^{(k)}+c_{1}\left(\frac{\rho_{a i, j}}{\rho_{c i, j}^{(k)}}-1\right) \rho_{i, j}^{(k)}, \\
& h_{i, j}^{(k+1)}=h_{i, j}^{(k)}+c_{2}\left(\frac{h_{a i, j}}{h_{c i, j}^{(k)}}-1\right) h_{i, j}^{(k)} .
\end{aligned}
$$

Equation (10) is the iterative formula for the improved ZohdyOldenburg direct inversion and gives resistivity inversion of 
the model. In this formula, $\rho_{i, j}^{(k)}$ stands for the true resistivity (or called intrinsic resistivity) of $k$ th model at $i$ th sounding point, of $j$ th layer; $\rho_{a i, j}$ the measured apparent resistivity at $i$ th sounding point, $j$ th frequency point; $\rho_{c i, j}^{(k)}$ the theoretical apparent resistivity of $k$ th model at $i$ th sounding point, $j$ th frequency point; in this paper the correction factor $c_{1}=0.5$.

Equation (11) is the iterative formula for the improved Oldenburg's iterative inversion and gives model depth inversion. In this formula, $h_{i, j}^{(k)}$ represents the depth of $k$ th model at $i$ th sounding point, of $j$ th layer; $h_{a i, j}$ the apparent depth at $i$ th sounding point, of $j$ th layer (calculated from Bostick's inversion formula); $h_{c i, j}^{(k)}$ the theoretical apparent depth of $k$ th model at $i$ th sounding point, of $j$ th layer (calculated from Bostick's inversion formula); in this paper the correction factor $c_{2}=0.5 c_{1}$.

3.2. Two-Dimensional Direct Inversion with Phase Information. Zhang and Xu [14] and Weidelt [12], 1972, derived the approximate relationship between MT apparent resistivity and impedance phase:

$$
\varphi(f) \approx \frac{\pi}{4}\left(1+\frac{d \lg \rho_{a}(f)}{d \lg f}\right) .
$$

In this relationship, $f$ represents the frequency, $\varphi(f)$ the impedance phase, and $\rho_{a}(f)$ the apparent resistivity.

If $D(f)=d \lg \rho_{a}(f) / d \lg f$, then

$$
D(f) \approx \frac{4}{\pi} \varphi(f)-1 .
$$

Bostick inversion formula is already known, so we get

$$
\rho(f)=\rho_{a}(f) \frac{1-D(f)}{1+D(f)}
$$

where $\rho(f)$ stands for the true resistivity in Bostick inversion formula.

When we plug (13) into (14), we obtain the inversion formula of impedance phase:

$$
\rho(f)=\rho_{a}(f)\left(\frac{\pi}{2 \varphi(f)}-1\right) .
$$

When small changes of impedance phase bring changes to resistivity, namely, both sides of (15) take a derivative with respect to $\varphi(f)$, the result is

$$
\frac{d \rho(f)}{d \varphi(f)}=\rho_{a}(f) \frac{\pi}{2} \frac{-1}{\varphi(f)^{2}}
$$

Ignoring the local minimum of differential, we find that (16) turns into

$$
\Delta \rho(f)=\rho_{a}(f) \frac{\pi}{2} \frac{-\Delta \varphi(f)}{\varphi(f)^{2}} .
$$

If we plug (17) into (10), the iterative formula of Zohdy's direct inversion, we get

$$
\rho_{i, j}^{(k+1)}=\rho_{i, j}^{(k)}+c_{1}\left(\frac{\rho_{a i, j}}{\rho_{c i, j}^{(k)}}-1\right) \rho_{i, j}^{(k)}+c_{1} \rho_{a i, j} \frac{\pi}{2} \frac{\varphi_{c i, j}^{(k)}-\varphi_{a i, j}}{\varphi_{a i, j}} .
$$

Equation (18) is the improved iterative formula with phase information for Zohdy's direct inversion. In this equation, $\varphi_{a i, j}$ stands for the measured impedance phase at $i$ th sounding point, $j$ th frequency point; $\varphi_{c i, j}^{(k)}$ the theoretical impedance phase of $k$ th model at $i$ th sounding point, $j$ th frequency point.

The model depth inversion still uses (11), the improved iterative formula for Oldenburg direct inversion. If we put (18) and (11) in the simultaneous form, then we will obtain the 2D MT improved Zohdy-Oldenburg direct inversion with phase information.

3.3. Calculation Method of Direct Inversion. The improved Zohdy-Oldenburg direct inversion is based on the direct inversion of Zohdy and Oldenburg, and it constructs the initial model of inversion with Bostick inversion formula and combines the formulas of Zohdy and Oldenburg simultaneously while it inverses the model resistivity and model depth. This method, featuring faster speed, higher precision, and faster convergence, can tackle a series of problems caused by solving large sparse Jacobian matrix.

Process of the improved Zohdy-Oldenburg direct inversion is as follows.

Step 1. Use Bostick inversion formula to calculate Bostick true resistivity and layer depth of each electric horizon, and fill 2D initial prediction model in the mesh model and perform a forward computation is as follows.

Step 2. Compare the measured apparent resistivity with the theoretical apparent resistivity obtained from the forward computation to check whether the precision can meet requirements. If the answer is yes, then it is certain that the prediction model is the result of the inversion.

Step 3. If not, modify the model parameters with ZohdyOldenburg iterative formula and obtain a new 2D prediction model. Conduct Bostick inversion, and get true resistivity and layer depth of each electric horizon. Refill the new 2D prediction model in the mesh model and again perform a $2 \mathrm{D}$ forward computation.

\section{Step 4. Return to Step 2.}

The fitting error between the measured apparent resistivity with the theoretical apparent resistivity will adopt mean square error; that is to say,

$$
\operatorname{Error}^{(k)}=\sqrt{\frac{1}{\mathrm{NP} \times N} \sum_{i=1}^{\mathrm{NP}} \sum_{j=1}^{N}\left(\frac{\rho_{a i, j}-\rho_{c i, j}^{(k)}}{\rho_{a i, j}}\right)^{2}},
$$




\begin{tabular}{|c|c|}
\hline$\rho=100 \Omega \cdot \mathrm{m}$ & $500 \mathrm{~m}$ \\
\hline$\rho=10 \Omega \cdot \mathrm{m}$ & $500 \mathrm{~m}$ \\
\hline
\end{tabular}

FIgURE 1: H-type MT cross-sectional model.

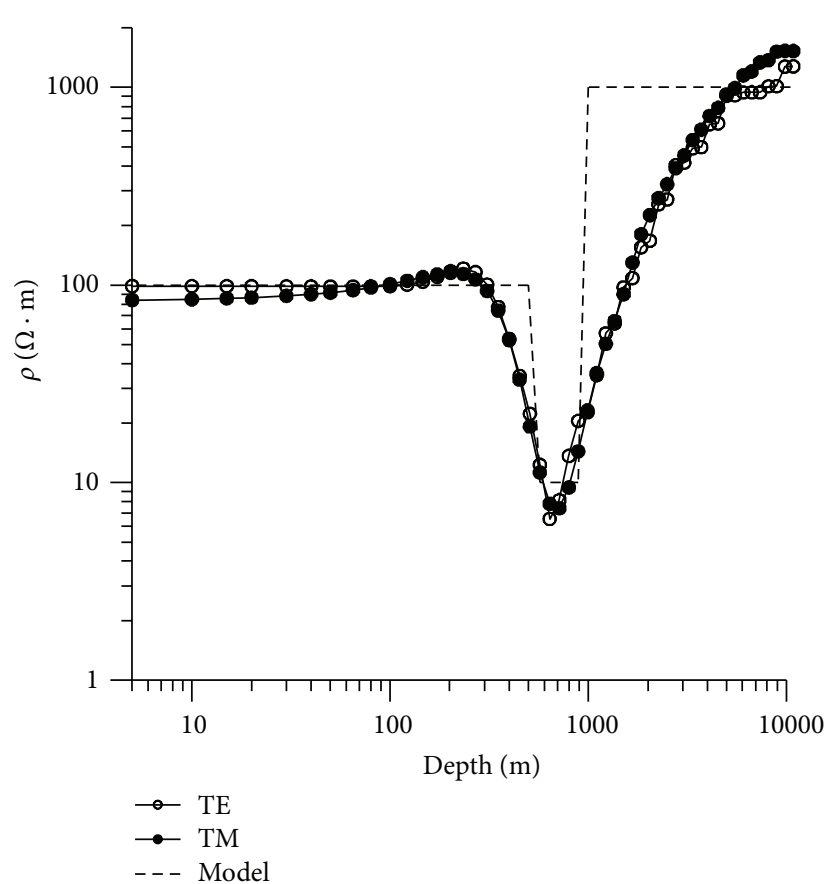

(a) Inversion result of apparent resistivity

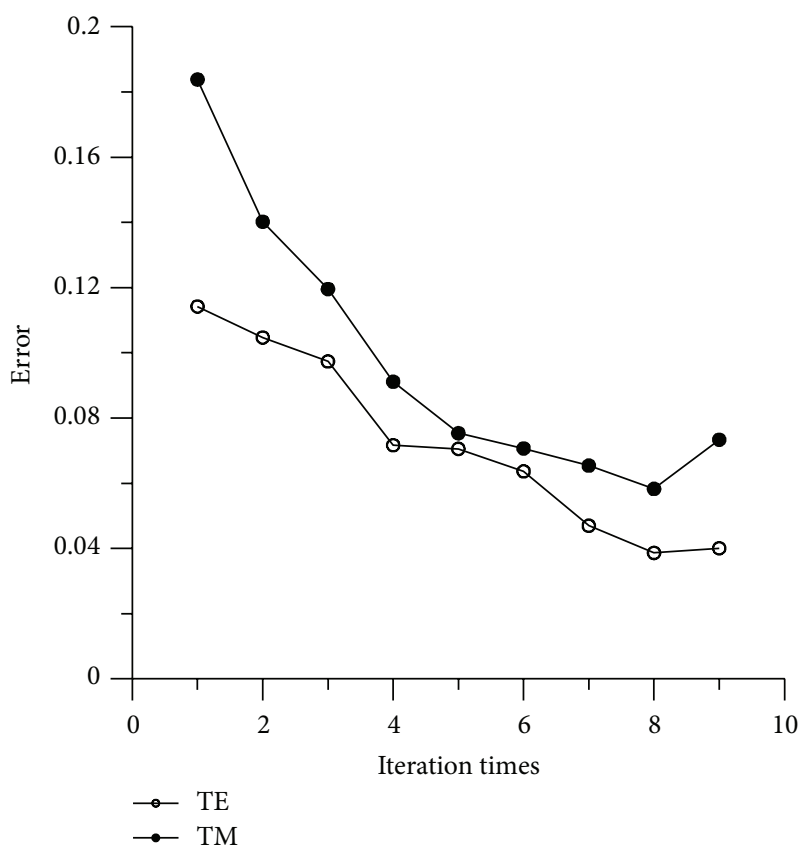

(b) Inversion fitting error

FIGURE 2: Inversion result.

where Error ${ }^{(k)}$ stands for the $k$ th prediction model's mean square error between the measured apparent resistivity and the theoretical apparent resistivity, NP the number of measuring points, and $N$ the number of frequencies.

\section{Analysis of Examples}

4.1. One-Dimensional Layered MT Model. Figure 1 is the Htype MT cross-sectional model of horizontally layered earth (3 layers), which is nonproportional and for illustration only. The resistivity and the layer thickness of the first layer are, respectively, $100 \Omega \cdot \mathrm{m}$ and $500 \mathrm{~m}$; for the second layer, they are $10 \Omega \cdot \mathrm{m}$ and $500 \mathrm{~m}$; the third layer's resistivity is $1000 \Omega \cdot \mathrm{m}$. The computation domain is $0 \sim 4 \mathrm{~km}$, the dot pitch is $100 \mathrm{~m}$, and it totaled 41 measuring points. The range of frequency is $10^{3} \sim 10^{-3} \mathrm{~Hz}$, it totaled 61 frequency points, and the sampling interval is base-10 logarithms.

Figure 2(a) is the inversion result of apparent resistivity, in which the horizontal axis represents depth (depth, unit: $\mathrm{m}$ ) while the vertical axis represents resistivity $(\rho$, unit: $\Omega \cdot \mathrm{m})$; Figure 2(b) is the curve graph of inversion fitting error, in which the horizontal axis represents iteration times and the vertical axis represents fitting error.
As can be seen from the inversion result of TE mode and TM mode, the anomalies of $\mathrm{H}$-type stratum are revealed clearly. In the TE mode inversion result, the iteration times are 9 , fitting errors percentage is $3.9 \%$, and it takes $623 \mathrm{~s}$; as for the TM mode inversion result, the iteration times are 9, fitting errors percentage is $6.2 \%$, and it takes $410 \mathrm{~s}$. The maximum relative error between these 2 modes is $5.3 \%$ and the mean square error is less than $2 \%$.

4.2. Rugged Topography 2D Structural Model. Figure 3 is the rugged topography $2 \mathrm{D}$ structural model. The largest drop in the rugged topography is $500 \mathrm{~m}$, which contains faults and high-resistivity and low-resistivity layers; the measuring points cover $0 \sim 20 \mathrm{~km}$, the dot pitch is $200 \mathrm{~m}$, and it totaled 101 measuring points; the frequency is within the common frequency spectrum of a V8 electrical prospecting apparatus, $10^{2.5} \sim 10^{-2.96} \mathrm{~Hz}$, the sampling interval is base- 10 logarithms, and it totaled 38 frequency points.

Figure 4 shows the cross-sectional view of inversion results, in which the horizontal axis represents measuring point location ( $X$, unit: $\mathrm{km}$ ) and the vertical axis depth (Depth, unit: km); Figures 4(a) and 4(b) are apparent resistivity inversion results of TE mode and TM mode, respectively, 


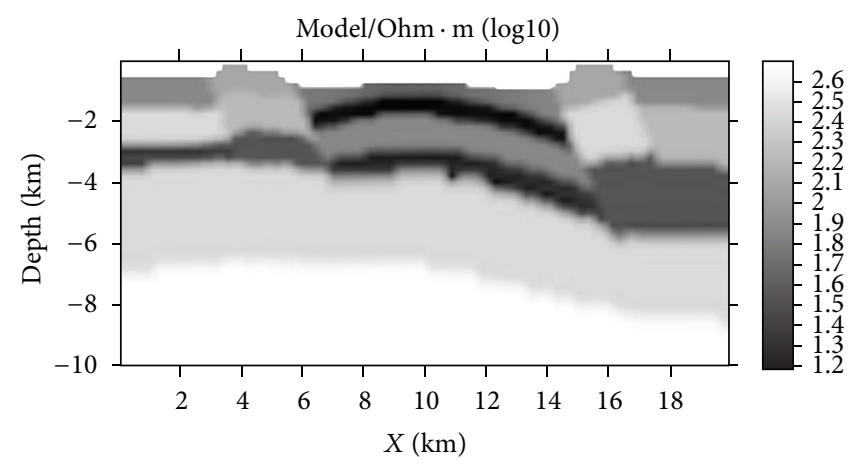

FIGURE 3: Rugged topography 2D structural model.

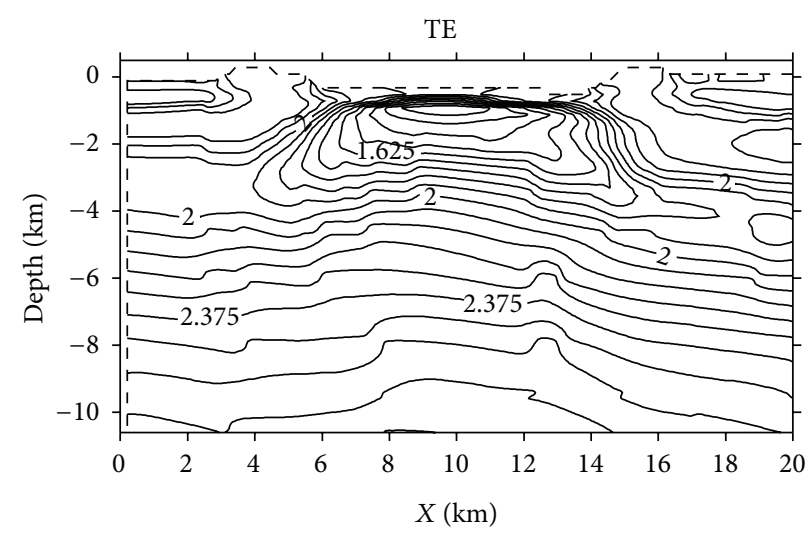

(a) Cross-sectional view of TE mode inversion result

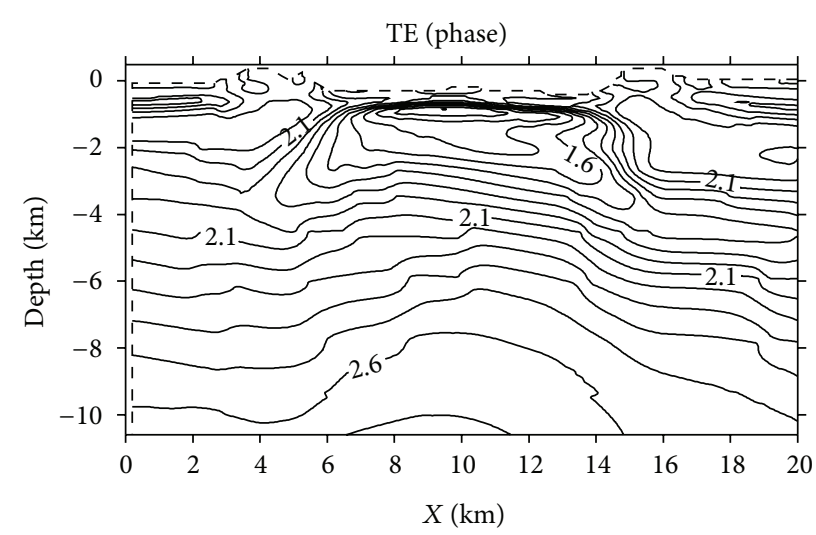

(c) Cross-sectional view of TE mode inversion result (with phase)

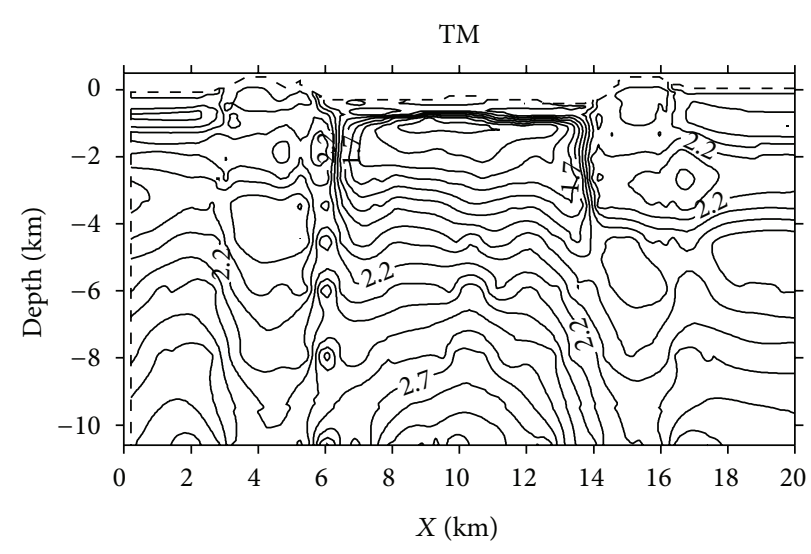

(b) Cross-sectional view of TM mode inversion result

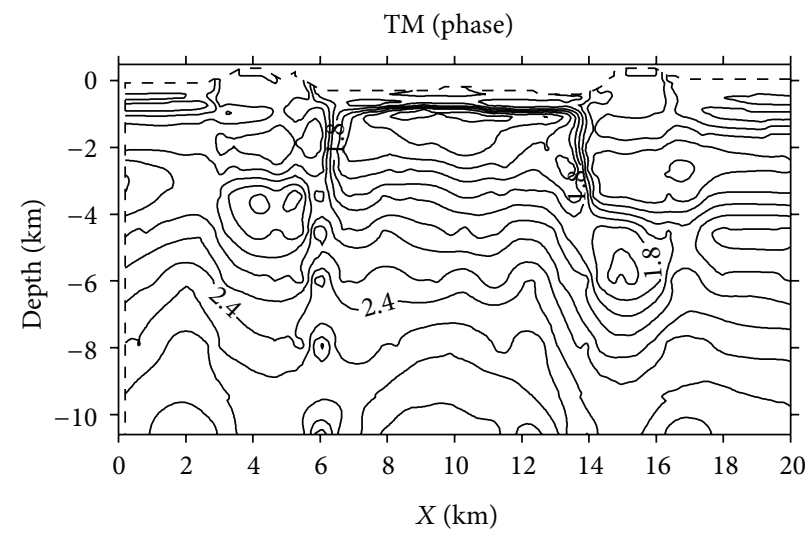

(d) Cross-sectional view of TM mode inversion result (with phase)

FIGURE 4: Cross-sectional view of inversion results.

while Figures 4(c) and 4(d) are apparent resistivity inversion results of TE mode and TM mode with phase. Figure 5 is the fitting error curve of inversion iteration, in which the horizontal axis represents number of iterations or iteration times, while the vertical axis represents fitting errors. Figure 5(a) is the comparison curve of fitting error of TE mode inversion iteration with and without phase while Figure 5(b) is the comparison curve of fitting error of TM mode inversion iteration with and without phase.
From the above cross-sectional view of inversion results, it can be seen that the impact of rugged topography is eliminated, and inversions with topography of TE mode and TM mode are able to reflect locations and anomalies of faults and high-resistivity and low-resistivity layers. But it fails to reflect the second low-resistivity layer in the middle area of the figure, due to the equivalence phenomenon in geophysics.

In the TE mode inversion without phase, the iteration times are 15 and the fitting error is $6.2 \%$. In the TE mode 


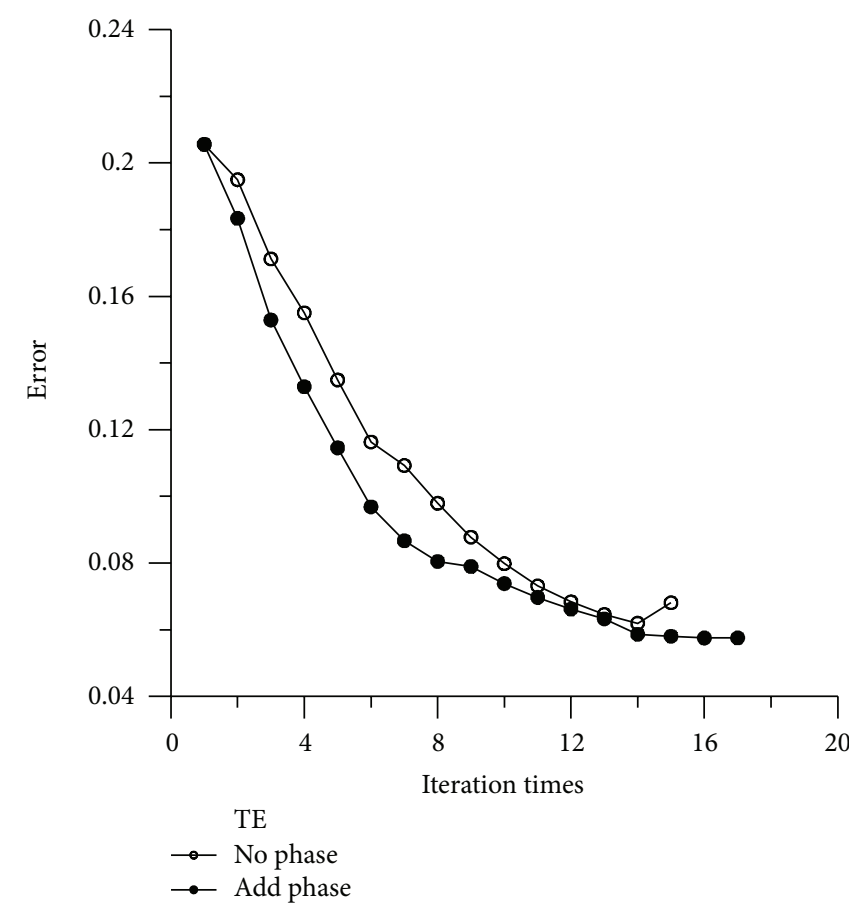

(a) Comparison curves of fitting error of TE mode inversion iteration with and without phase

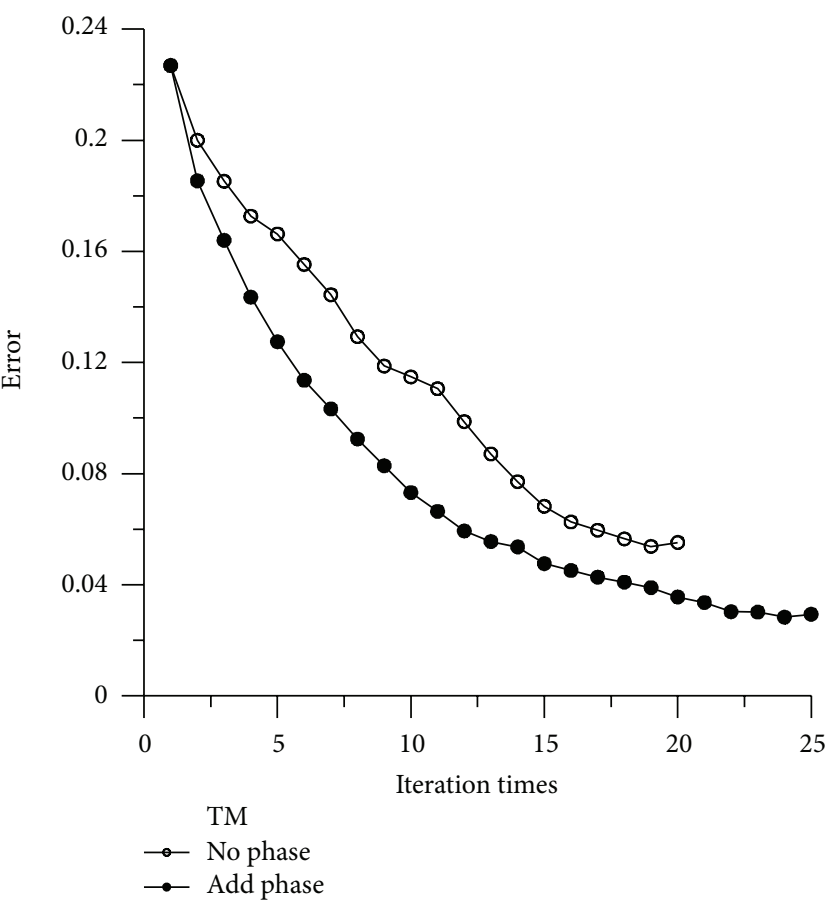

(b) Comparison curves of fitting error of TM mode inversion iteration with and without phase

FIGURE 5: Fitting error curve of inversion iteration.

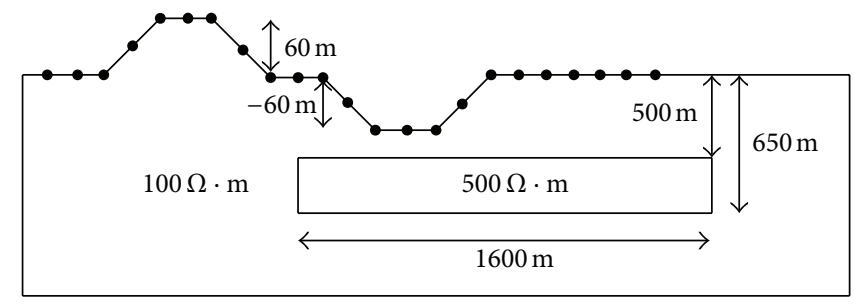

FIGURE 6: Geoelectric cross section model with abnormal body under rugged topography.

inversion with phase, the iteration times are 17 and the fitting error is $5.7 \%$. In the TM mode inversion without phase, the iteration times are 20 and the fitting error is 5.3\%. In the TM mode inversion with phase, the iteration times are 25 and the fitting error is $2.8 \%$.

\subsection{Model with Abnormal Body under Rugged Topography.} Figure 6 shows the model with abnormal body under rugged topography, which is nonproportional and just for the convenience of easier understanding. In this figure, the drop is $60 \mathrm{~m}$, the background resistivity is $100 \Omega \cdot \mathrm{m}$, and it contains a high-resistivity abnormal body of $500 \Omega \cdot \mathrm{m}$, which is $1600 \mathrm{~m}$ long and $150 \mathrm{~m}$ high, and it is $500 \mathrm{~m}$ away from its upper top surface to the ground surface. The measuring points cover $0 \sim 4 \mathrm{~km}$, the dot pitch is $100 \mathrm{~m}$, and it totaled 41 measuring points; the frequency is $10^{4} \sim 10^{-2} \mathrm{~Hz}$, the sampling interval is base-10 logarithms, and it totaled 61 frequency points.

Figure 7 is the cross section of the inversion result. It is a resistivity (or intrinsic resistivity) contour map ( $\rho$, unit: $\Omega \cdot \mathrm{m}$ ), in which the horizontal axis stands for measuring points location ( $X$, unit: $\mathrm{km}$ ) and the vertical axis depth (Depth, unit: $\mathrm{km}$ ). Figures $7(\mathrm{a})$ and $7(\mathrm{~b})$ are apparent resistivity inversion results of TE mode and TM mode, respectively, while Figures 7(c) and 7(d) are apparent resistivity inversion results of TE mode and TM mode with phase. Figure 8 is the fitting error curve of inversion iteration, in which the horizontal axis represents number of iteration times, while the vertical axis represents fitting errors. Figure $8(a)$ is the comparison curve of fitting error of TE mode inversion iteration with and without phase while Figure $8(\mathrm{~b})$ is the comparison curve of fitting error of TM mode inversion iteration with and without phase.

The key of inversions with topography lies in the reliability of numerical modeling, which is influenced by topography, in forward calculation. As can be observed from the cross-sectional view of the inversion results, the impact of rugged topography is significantly reduced in the TE mode and the TM mode. And since the impact of topography on 


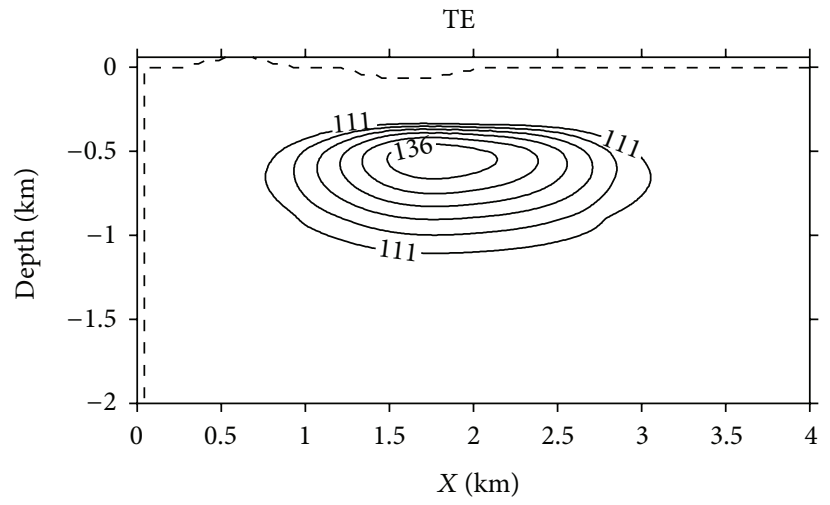

(a) Cross-sectional view of TE mode inversion result

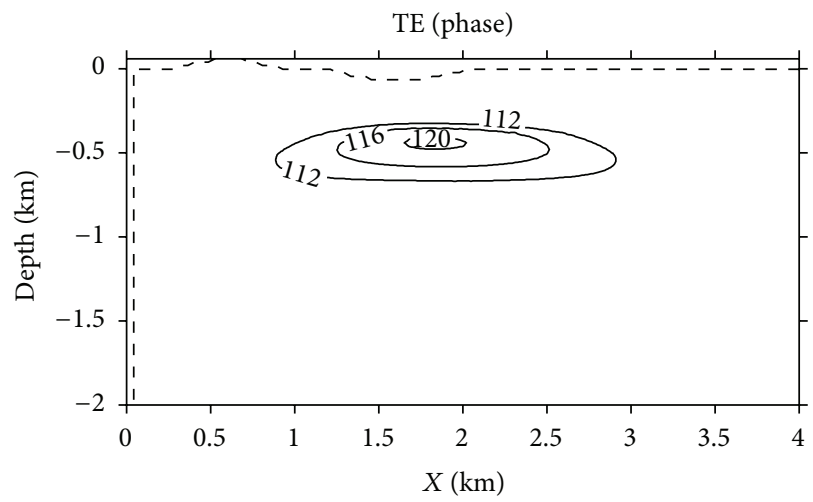

(c) Cross-sectional view of TE mode inversion result (with phase)

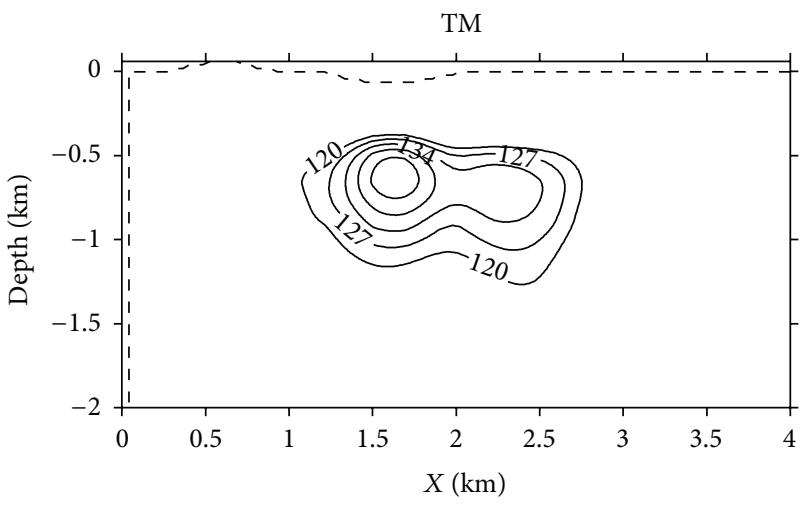

(b) Cross-sectional view of TM mode inversion result

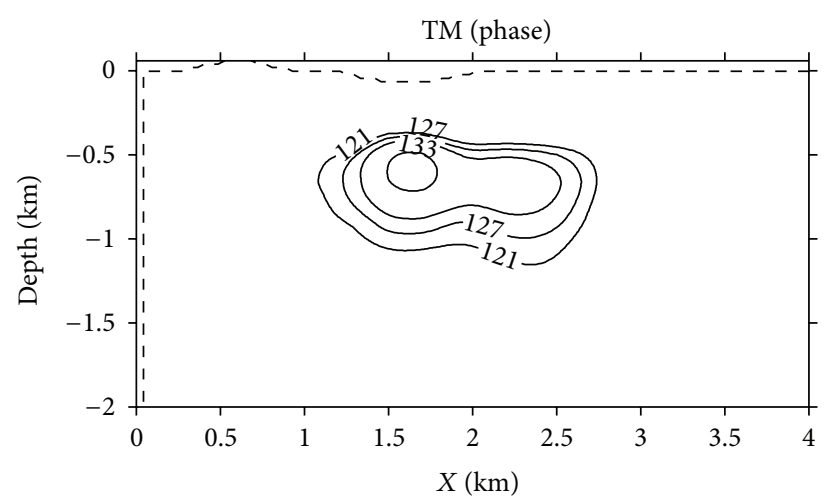

(d) Cross-sectional view of TM mode inversion result (with phase)

FIGURE 7: Cross-sectional view of inversion results.

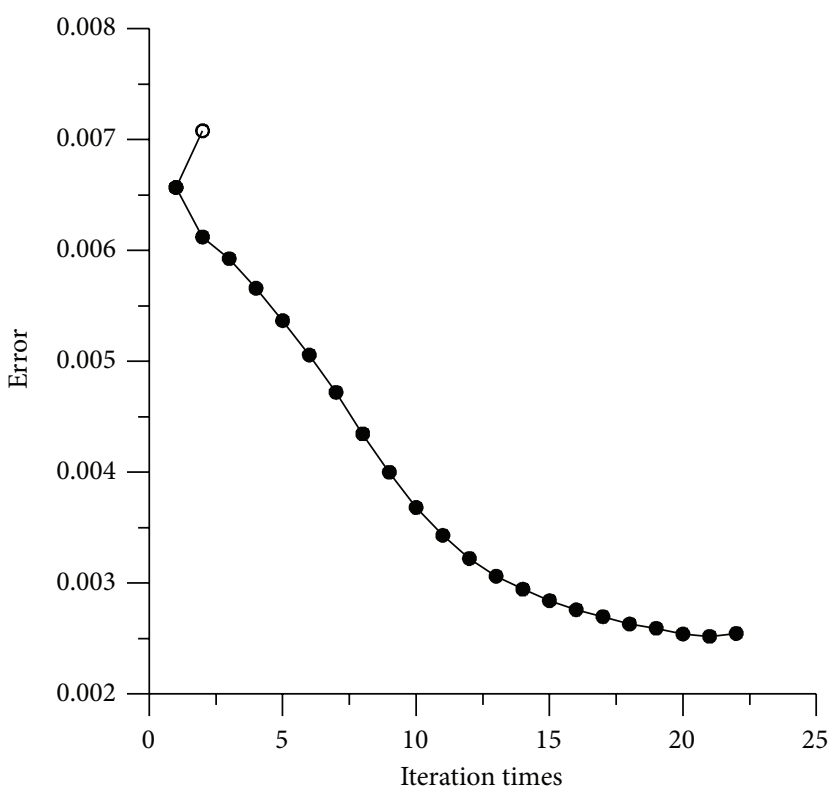

TE

$$
\begin{aligned}
& \because \text { No phase } \\
& \rightarrow \text { Add phase }
\end{aligned}
$$

(a) Comparison curves of fitting error of TE mode inversion iteration with and without phase

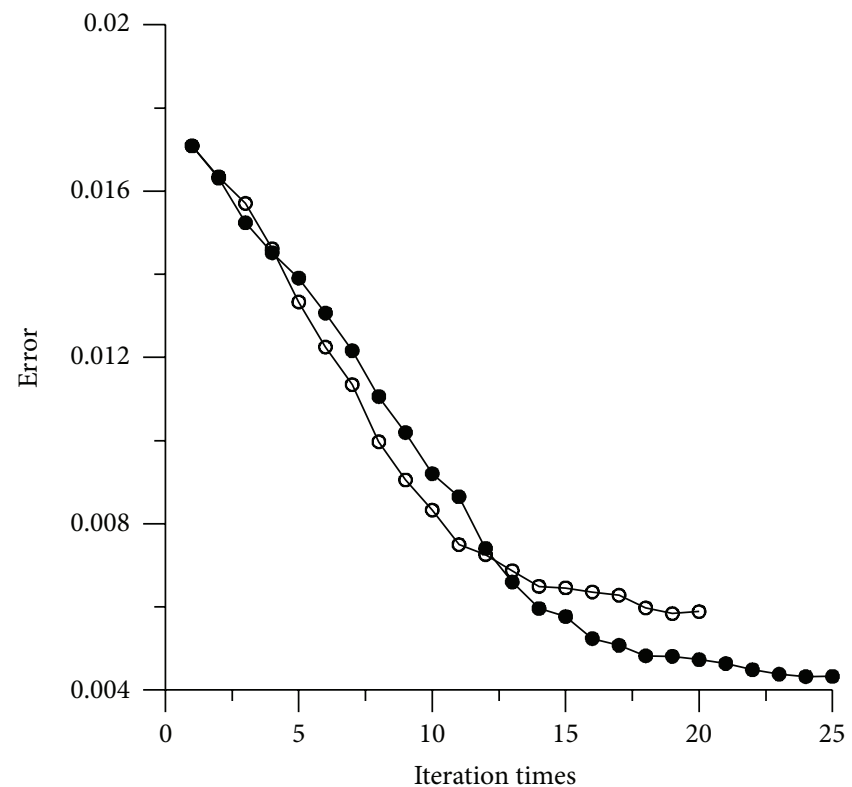

TM

$\rightarrow$ No phase

$\rightarrow$ Add phase

(b) Comparison curves of fitting error of TM mode inversion iteration with and without phase

FIGURE 8: Fitting error curve of inversion iteration. 
TE mode is relatively small, the inversion result of abnormal body of TE mode is better than that of TM mode. In the TE mode inversion without phase, the iteration times are 2 and the fitting error is $6.6 \%$; in the TE mode inversion with phase, the iteration times are 22 and the fitting error is $0.25 \%$. In the TM mode inversion without phase, the iteration times are 20 and the fitting error is $0.58 \%$; in the TM mode inversion with phase, the iteration times are 25 and the fitting error is $4.3 \%$.

\section{Conclusions}

In this paper, the proposed rugged topography 2D MT improved Zohdy-Oldenburg direct inversion is a asymptotic iteration method based on the least square, so the modification direction of this model always points to the descent direction of the objective function. This method embodies the features of both Zohdy's asymptotic iteration method (ratio method) and Oldenburg's asymptotic iteration method (difference method). The advantages of faster convergence and higher precision bypass large calculations of the Jacobian matrix and large sparse linear systems of equations and enable direct modifications and comparisons of the model parameters. In contrast with the conventional linear inversion, the calculation speed of the new method in this paper can be increased by more than 10 times.

\section{References}

[1] G. A. Newman and D. L. Alumbaugh, "Three-dimensional magnetotelluric inversion using non-linear conjugate gradients," Geophysical Journal International, vol. 140, no. 2, pp. 410-424, 2000.

[2] W. Rodi and R. L. Mackie, "Nonlinear conjugate gradients algorithm for 2-D magnetotelluric inversion," Geophysics, vol. 66, no. 1, pp. 174-187, 2001.

[3] C. Lin, H. Tan, and T. Tong, "Parallel rapid relaxation inversion of 3D magnetotelluric data," Applied Geophysics, vol. 6, no. 1, pp. 77-83, 2009.

[4] W. Siripunvaraporn, "Three-dimensional magnetotelluric inversion: an introductory guide for developers and users," Surveys in Geophysics, vol. 33, no. 1, pp. 5-27, 2012.

[5] A. A. R. Zohdy, "A new method for the automatic interpretation of Schlumberger and Wenner sounding curves," Geophysics, vol. 54, no. 2, pp. 245-253, 1989.

[6] D. W. Oldenburg and R. G. Ellis, "Inversion of geophysical data using an approximate inverse mapping," Geophysical Journal International, vol. 105, no. 2, pp. 325-353, 1991.

[7] X. Shizhe and L. Bin, "The curve comparison method of MT inversion for one-dimensional continuous medium," Acta Geophysica Sinica, vol. 38, no. 5, pp. 676-682, 1995 (Chinese).

[8] L. Bin and X. Shizhe, "The curve comparison method of one-dimensional inversion for electrical sounding," Computing Techniques for Geophysical and Geochemical Exploration, vol. 17, no. 1, pp. 23-27, 1995 (Chinese).

[9] R. Baiyao, Asymptotic Inversion of DC Resistivity Sounding and Numberical Modeling of 2-D Time Domain Electromagnetic Response, Ocean University of Qingdao, 1995, Chinese.

[10] R. Baiyao and X. Shizhe, "Rapid inversion of two-dimensional DC resistivity sounding curve," Geophysical and Geochemical Exploration, vol. 20, no. 6, pp. 455-460, 1996 (Chinese).
[11] R. Baiyao, X. Shizhe, D. Shikun et al., "Rapid inversion of 2-D magnetotelluric sounding date," Journal of Guilin University of Technology, vol. 18, no. 1, pp. 53-56, 1998 (Chinese).

[12] P. Weidelt, "The inverse problem of geomagnetic induction," Journal of Geophysics, vol. 38, no. 1, pp. 257-289, 1972 (German).

[13] D. Zhang and S. Xu, "One-dimensional magnetotelluric curve comparison inversion with phase information," Seismology and Geology, vol. 23, no. 2, pp. 227-231, 2001 (Chinese).

[14] D. Zhang and S. Xu, "A feasibility study of rapid twodimensional MT curve comparison inversion," Seismology and Geology, vol. 23, no. 2, pp. 232-237, 2001 (Chinese). 


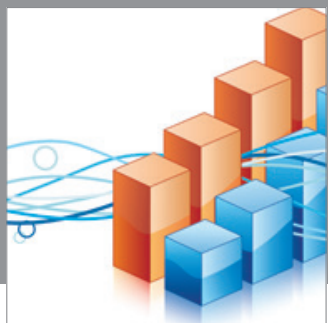

Advances in

Operations Research

mansans

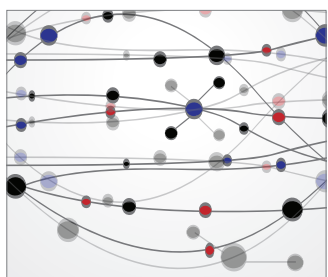

The Scientific World Journal
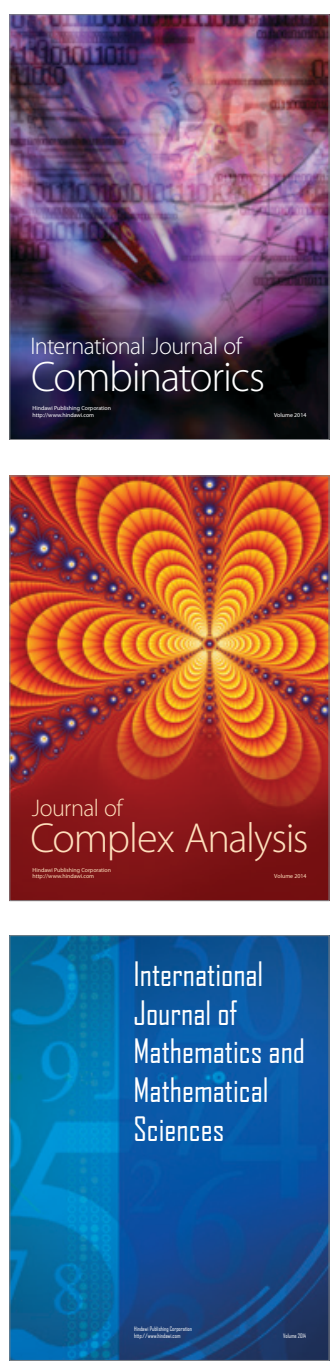
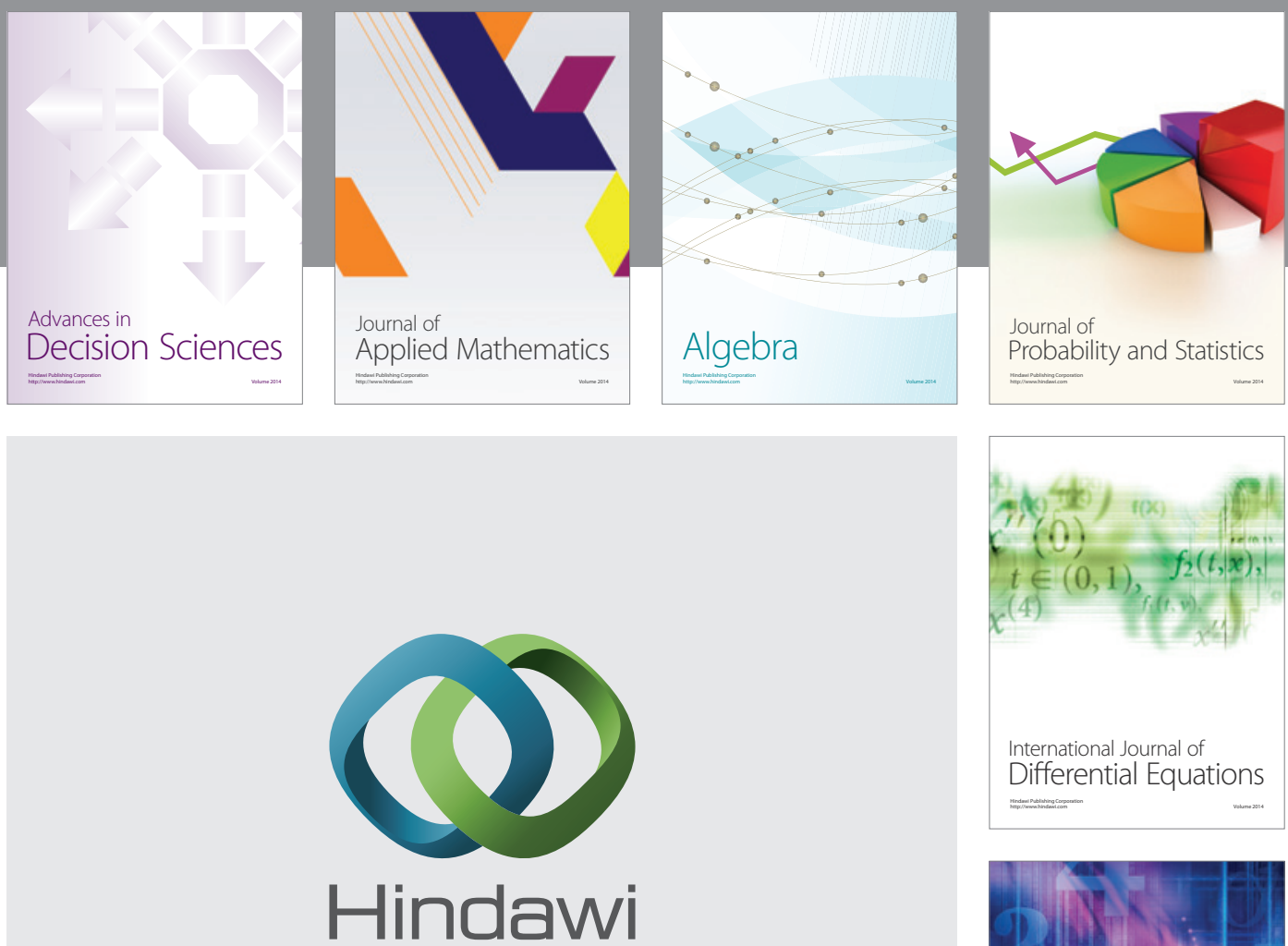

Submit your manuscripts at http://www.hindawi.com
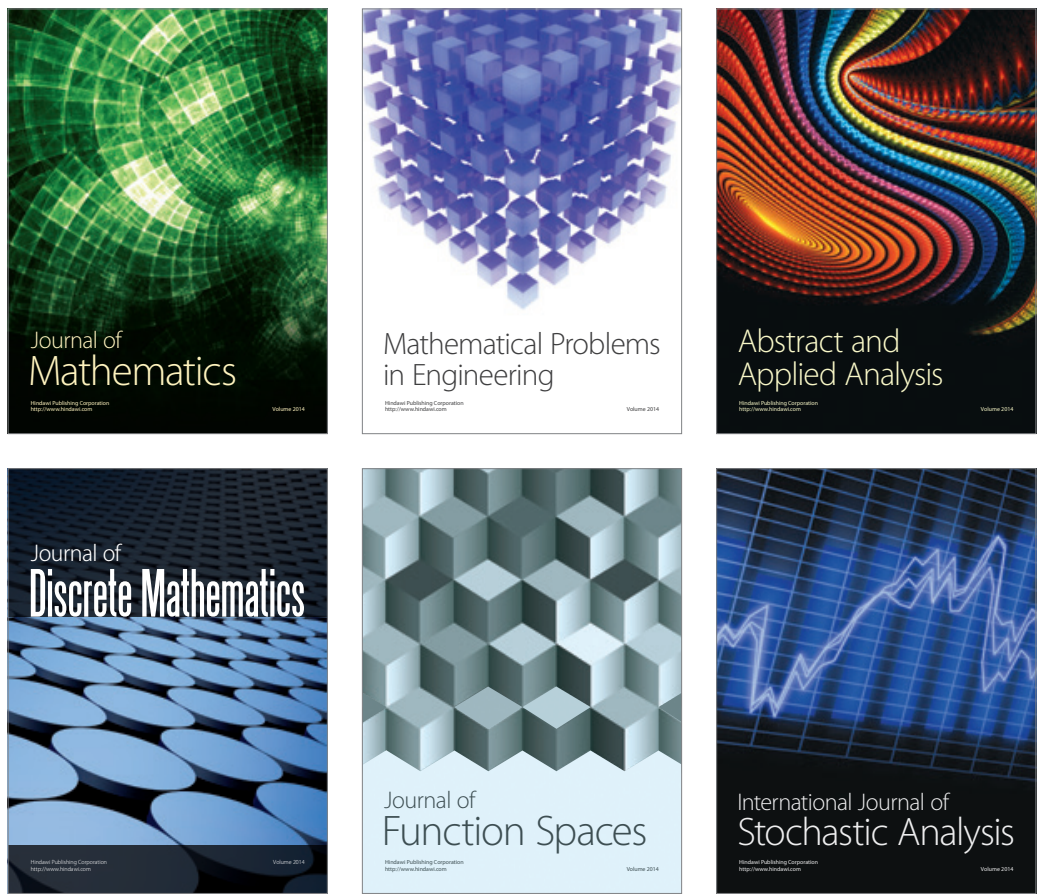

Journal of

Function Spaces

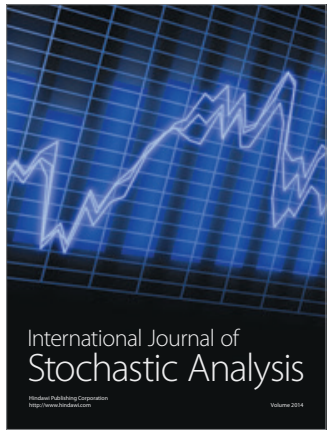

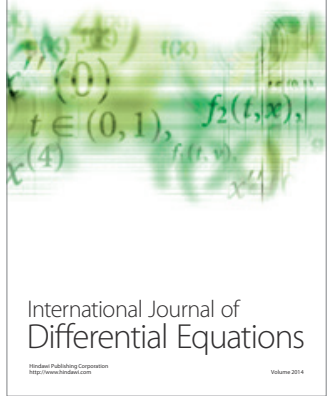
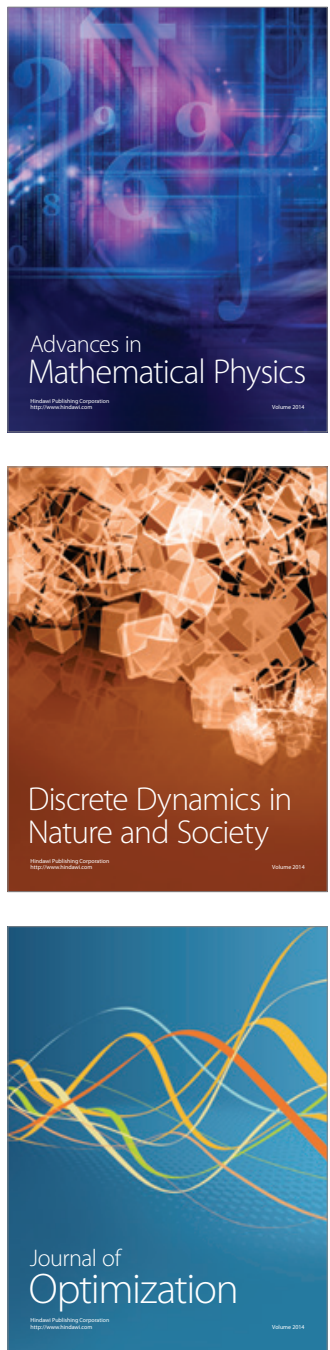